\title{
On Loss Probabilities in Presence of Redundant Packets with Random Drop
}

\author{
Parijat Dube Omar Ait-Hellal Eitan Altman *
}

\begin{abstract}
The purpose of this paper is to study the loss probabilities of messages in an $\mathrm{M} / \mathrm{M} / 1 / \mathrm{K}$ queueing system where in addition to losses due to buffer overflow there are also random losses in the incoming and outgoing links. We focus on the influence of adding redundant packets to the messages (as in error correction coding e.g. Reed-Solomon code etc.). In the first part we use multi-dimensional probability generating functions for solving the recursions which generalize those introduced by Cidon, Khamisy and Sidi [5] for computing the loss probabilities and derive analytical formulae for a special case. In the second part of the paper we use combinatorial arguments and Ballot theorem results to alternatively obtain the loss probabilities. The analytical results allow us to investigate when does adding redundancy decrease the loss probabilities.
\end{abstract}

Keyword: queueing analysis, forward error correction, Poisson process, loss probabilities, generating functions, Ballot theorems.

\section{Introduction}

The loss probability of packets in queueing networks is an important performance measure in telecommunication networks and some other applications. Rapid progress in the development of fiber optics allows to achieve a bit error rate of $10^{-14}$; information loss is then essentially due to congested nodes and buffer overflow. However, in wireless networks random losses of packets also occur in the channels/links apart from congestion losses.

Often, when a message is divided into several packets, the loss of one packet results in the loss of the whole message. In order to reduce the loss probabilities, one may add redundant packets, so that lost packets can often be reconstructed. Indeed, there exist erasure recovery codes that, by adding $k$ redundant packets to a message, enable to reconstruct up to $k$ losses (see [5],[7],[10], [9] and references therein). We note, however, that by adding redundant packets, the workload increases and thus the loss probability of a packet may increase ([1]).

${ }^{*}$ Parijat Dube and Eitan Altman are with Project MISTRAL, INRIA, 2004, Route des Lucioles, B.P.93,06902, Sophia Antipolis Cedex, France. Parijat.Dube, Eitan.Altman@sophia.inria.fr. Omar Ait-Hellal is with Xbind Incorporation, 55 Broad Street, New York, NY 10004, U.S.A. oaithel@xbind.com 
Alternatively, if redundancy is added in such a way that the total workload remains unchanged then this will result in a decrease in the throughput of useful information transmitted by the source. Thus there are two types of tradeoffs to be studied (according to whether we want to keep the total transmitted throughput the same, or only the throughput corresponding to useful transmitted information).

In this paper we are concerned with studying the loss probabilities of messages in queueing systems where in addition to losses due to buffer overflow there are also random losses on the incoming and outgoing links to the bottleneck node. In particular, we study the tradeoffs mentioned in the previous paragraph.

The problem of analyzing loss probabilities due to congestion losses in the presence of redundant packets has been addressed in earlier works $[7,5,4,9]$. In [7], the authors have used an approximation based on the assumption of independence between consecutive losses, and have shown that redundancy results in a decrease of loss probabilities by 10 to 100 . Exact numerical methods based on recursions in [5] led, to an opposite conclusion, i.e., adding redundancy causes an increase in the loss probabilities. Explicit expressions for the losses have then been developed in $[4,9]$ and references therein which allowed to obtain regions of parameters in which Forward Error Correction (FEC) ${ }^{1}$ is useful. In particular, in [4] information theoretical type of channel capacity has been obtained for channels with congestion losses (and general service and inter-arrival times). All these references studied models where losses are only due to congestion. Such models are useful in fiber-optic networks, where the main source of losses in the network is indeed overflow of the bottleneck buffer(s). There are however other situations in which non-negligible amount of losses may also occur at the links, such as in wireless and in satellite communications.

The goal of this paper is therefore to determine the role of redundant packets in networks in which losses may be due to both phenomena: link losses (which we call random losses) and losses due to buffer overflow (which we call congestion losses). We obtain expressions that permit us to study two scenarios for adding FEC. In the first, we assume that the global transmission rate is unchanged, so that when adding FEC we reduce the rate of useful information. We then analyze how does the received rate of useful information depend on the FEC. In the second scenario we keep the rate of useful information unchanged; adding FEC then increases the congestion and hence the losses, but on the other hand allows one to recover some lost packets. We note that not only is our model a generalization of the previous work in considering both congestion as well as random losses, but also the first scenario that we investigate has not been considered earlier even in the context of congestion losses only $[7,5,4,9]$.

The paper is structured as follows. In Sec. 2 we present our model and its motivation. In Sec. 3 we present our main results derived using an algebraic approach involving multidimensional generating functions; the proof is provided in Appendix. In Sec. 4 we provide numerical examples and discuss the region where adding redundancy improves the perfor-

\footnotetext{
${ }^{1}$ the technique of transmitting redundant information with original information is called FEC
} 


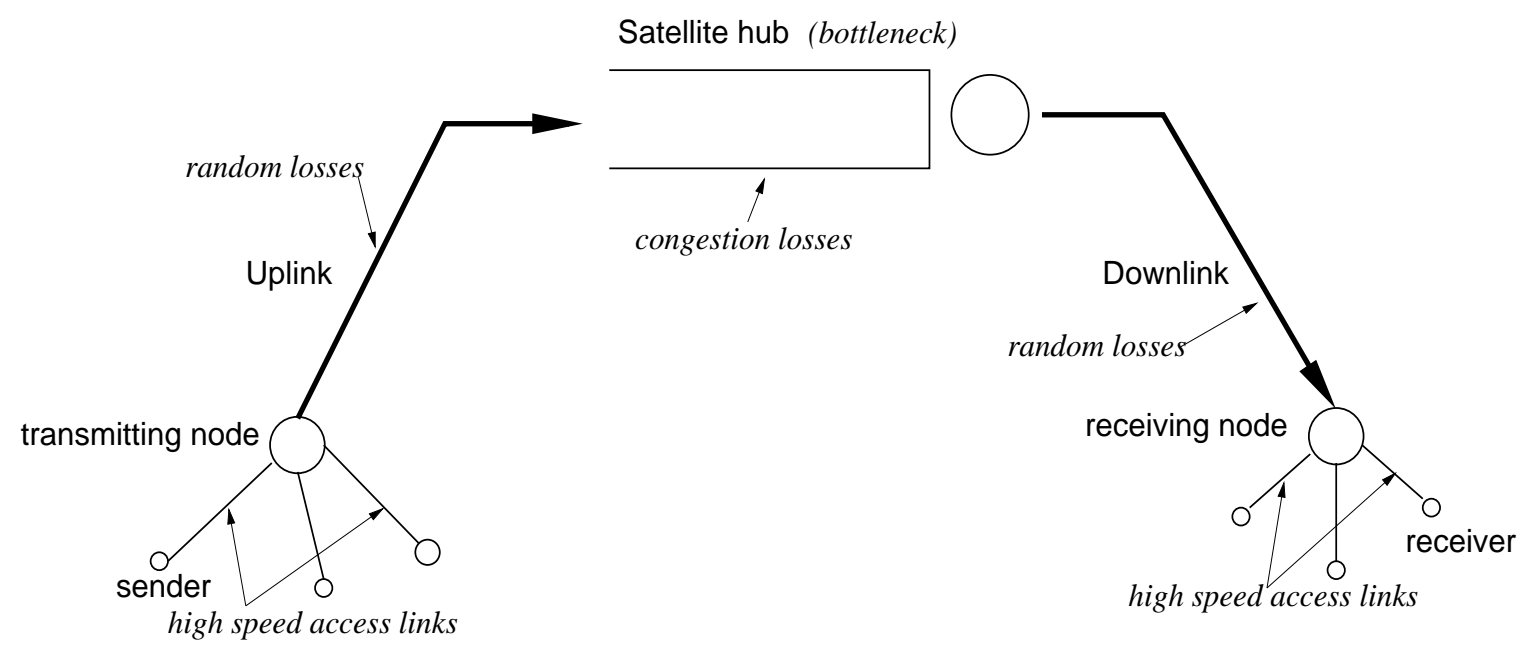

Figure 1: A motivational scenario: FEC for satellite communication

mance. In Sec. 5 we employ a combinatorial approach using Ballot theorems to obtain explicit expressions for loss probabilities employing techniques developed in [9]. Finally, we conclude in Sec. 6 with directions for further work.

\section{The Model and its Motivation}

We consider networks consisting of a noisy link (in which random losses occur) followed by a bottleneck buffer, or more generally, of a buffer that is in-between two noisy links. The latter is a suitable model for satellite connections (see Fig. 1) in which there is a noisy uplink and a noisy downlink connection with further losses that may be due to congestion inside the satellite (in which the buffer sizes are typically much smaller than in the terrestrial networks). We assume throughout that a packet that is corrupted before it arrives to the bottleneck queue is discarded and thus does not occupy any space in the buffer.

In the analysis below we shall model random losses in the incoming link (uplink) and congestion losses at the node. We consider an $\mathrm{M} / \mathrm{M} / 1$ queue with a finite buffer of size $K$ (including the packet in service). We assume that losses can be caused either by a buffer overflow or randomly with probability $r$ in the incoming link. The arrival process from the source is assumed to be Poisson with rate $\lambda$ and the service times of packets is exponentially distributed with rate $\mu$. Hence, the effective arrival process to the system (buffer) can be assumed to be Poisson with rate $\lambda_{e} \triangleq(1-r) \lambda$. Define $\bar{r} \triangleq 1-r, \rho \triangleq \lambda_{e} / \mu$, and $\rho_{r} \triangleq \rho / \bar{r}$. We present a recursive scheme for computing $P(j, n)$ which is the probability of $j$ losses (including random losses in the incoming link and congestion losses at the node) among $n$ consecutive packets in such a model. 
Remark 1 The case when there are losses in both the incoming and outgoing links can be analysed once we have $P(j, n)$. For example, let the random loss probability in the outgoing link be $u$ and let $\mathcal{P}_{j, n}$ be the probability of $j$ losses among $n$ consecutive packets of a message when there are random losses with probability $r$ in the incoming link, congestion losses due to buffer overflow at the node and random losses with probability $u$ in the outgoing link. Then

$$
\mathcal{P}_{j, n}=\sum_{w=0}^{j}\left(\begin{array}{c}
n-j+w \\
w
\end{array}\right) u^{w}(1-u)^{n-j} P(j-w, n) .
$$

Thus knowing $P(j, n)$, which is the loss probability in the model we consider (i.e., random losses in the incoming link and congestion losses at the node) one can obtain the loss probabilities for the case when random losses can occur both in the incoming and the outgoing links.

\section{Approach Using Generating Functions: Main Results}

For the system with Poisson arrivals with rate $\lambda_{e}$ and exponential transmission rate $\mu$, in steady state, the probability of finding $i$ packets in the system at an arbitrary epoch is given by

$$
\Pi(i)=\frac{\rho^{i}}{\sum_{l=0}^{K} \rho^{l}} .
$$

Define $Q_{i}(k)$ to be the probability that $k$ packets out of $i$ leave the system during an interarrival epoch. We have

$$
\begin{aligned}
Q_{i}(k) & =\rho \alpha^{k+1} \quad 0 \leq k \leq i-1 \\
Q_{i}(i) & =\alpha^{i}, \quad \text { where } \quad \alpha:=(1+\rho)^{-1} .
\end{aligned}
$$

Denote by $P_{i}^{a}(j, n)$ the probability of $j$ losses in a block of $n$ consecutive packets, given that there are $i$ packets in the system just before the arrival of the first packet in the block. Since the first packet in the block is arbitrary, we have

$$
P(j, n)=\sum_{i=0}^{K} \Pi(i) P_{i}^{a}(j, n) .
$$

The recursive scheme for computing $P_{i}^{a}(j, n)$ is then

$$
\begin{aligned}
& P_{i}^{a}(j, 1)=\left\{\begin{array}{ll}
\bar{r} & j=0 \\
r & j=1 \\
0 & j \geq 2,
\end{array} \quad i=0,1, \ldots, K-1\right. \\
& P_{K}^{a}(j, 1)= \begin{cases}1 & j=1 \\
0 & j=0, j \geq 2 .\end{cases}
\end{aligned}
$$


For $n \geq 2$ we have

$$
\begin{aligned}
P_{i}^{a}(j, n) & =\bar{r} \sum_{k=0}^{i+1} Q_{i+1}(k) P_{i+1-k}^{a}(j, n-1)+r \sum_{k=0}^{i} Q_{i}(k) P_{i-k}^{a}(j-1, n-1) \\
0 \leq i \leq K-1 & \\
P_{K}^{a}(j, n) & =\sum_{k=0}^{K} Q_{K}(k) P_{K-k}^{a}(j-1, n-1) .
\end{aligned}
$$

Next, we state the main results, whose detailed proofs are given in the next section. Define the probability generating function (pgf)

$$
q(y, z) \triangleq \sum_{j=0}^{\infty} \sum_{n=1}^{\infty} y^{j} z^{n-1} P(j, n) .
$$

Let $x_{1}(y, z)$ and $x_{2}(y, z)$ be the solutions in $x$ of $\left.x^{2}-(1+\rho-r \rho y z) x+\bar{r} \rho z\right)=0$ :

$$
\begin{aligned}
& x_{1}(y, z)=\frac{1+\rho-r \rho y z+\sqrt{(1+\rho-r \rho y z)^{2}-4 \bar{r} \rho z}}{2} \\
& x_{2}(y, z)=\frac{1+\rho-r \rho y z-\sqrt{(1+\rho-r \rho y z)^{2}-4 \bar{r} \rho z}}{2} .
\end{aligned}
$$

We shall often write simply $x_{1}$ and $x_{2}$ for $x_{1}(y, z)$ and $x_{2}(y, z)$. Define, for all $k \geq 1$, $\delta_{k}=x_{1}^{k}-x_{2}^{k}, \phi_{k}=(\bar{r}+r y) z \delta_{k-1}-\delta_{k}$. Let $R_{K}=\left(\sum_{l=0}^{K} \rho^{l}\right)^{-1}$.

Proposition 1 The pgf $q$ is given by

$$
\begin{aligned}
q(y, z)= & \frac{R_{K}}{1-(\bar{r}+r \rho y) z}\left[(\bar{r}+r y) R_{K-1}^{-1}+y \rho^{K}\right. \\
& \left.+z \rho(\alpha \rho)^{K}(\bar{r}(y-\alpha)-\alpha \rho y) A(y, z)+r z y(\alpha \rho)^{K} B(y, z)\right]
\end{aligned}
$$

where $A(y, z)$ and $B(y, z)$ solve

$$
\begin{gathered}
\left(\begin{array}{cc}
z \rho \alpha\left(\alpha x_{1}\right)^{K+1}\left(y\left(\bar{r}-\alpha x_{1}\right)-\bar{r} \alpha\right) & z \alpha^{2}\left(\bar{r}\left(x_{1}-\rho\right)+r x_{1} y\left(\alpha x_{1}\right)^{K}\right) \\
z \rho \alpha\left(\alpha x_{2}\right)^{K+1}\left(y\left(\bar{r}-\alpha x_{2}\right)-\bar{r} \alpha\right) & z \alpha^{2}\left(\bar{r}\left(x_{2}-\rho\right)+r x_{2} y\left(\alpha x_{2}\right)^{K}\right)
\end{array}\right)\left(\begin{array}{c}
A(y, z) \\
B(y, z)
\end{array}\right) \\
\quad=(-1)\left(\begin{array}{c}
\left(1-\alpha x_{1}\right) \alpha x_{1}^{K+1} y+\left(1-\alpha x_{1}\right) \alpha x_{1}(r y+\bar{r})\left(\frac{1-x_{1}^{K}}{1-x_{1}}\right) \\
\left(1-\alpha x_{2}\right) \alpha x_{2}^{K+1} y+\left(1-\alpha x_{2}\right) \alpha x_{2}(r y+\bar{r})\left(\frac{1-x_{2}^{K}}{1-x_{2}}\right)
\end{array}\right)
\end{gathered}
$$


For $y=0$, Proposition 1 simplifies to:

$$
q(0, z)=\bar{r} \frac{R_{K}}{1-\bar{r} z}\left[R_{K+1}^{-1}-z \rho^{K} A(0, z)\right] .
$$

Having obtained the probability generating function, the explicit expressions for the required probabilities can be obtained by inverting $q(y, z)$. In particular we shall focus on $P_{\rho}(>j, n)$, the probability of losing more than $j$ packets out of $n$. We investigate, in particular, the cases of $j=0,1$, in order to be able to decide whether adding a redundant packet to each message results in a decrease of the loss probability.

To stress the dependence of the different quantities (such as the pgf $q$ ) on the random loss parameter $r$ and on $\lambda$, we shall sometimes add $r$ and $\lambda$ explicitly to the notation as subscript (e.g. we shall write $q_{r}^{\lambda}(y, z)$ ). The next Corollary shows that there is a simple product form expression for the probability of no losses among $n$ consecutive packets. In this product, the first term corresponds to the probability of no random losses (in a system that has no congestion losses), and the second one corresponds to the probability of no congestion losses (in a system that has no random losses, and in which the arrival rate is reduced to $\bar{r} \lambda$ ).

Corollary 1 The following holds: (i) $q_{r}^{\lambda}(0, z)=q_{0}^{\bar{r} \lambda}(0, \bar{r} z) \bar{r}$, (ii) $P_{r}^{\lambda}(0, n)=\bar{r}^{n} P_{0}^{\bar{r} \lambda}(0, n)$.

\section{Proof:}

From (9) we have:

$$
q_{r}^{\lambda}(0, z)=\bar{r} \frac{R_{K}}{1-\bar{r} z}\left[R_{K+1}^{-1}-z \rho^{K} A_{r}^{\lambda}(0, z)\right]
$$

where $A_{r}^{\lambda}(0, z)=\frac{\left(1-\alpha x_{1}\right) \alpha x_{1} \frac{\left(1-x_{1}^{K}\right)}{\left(1-x_{1}\right)}}{z^{2} \bar{r} \alpha^{2}\left(\rho\left(\alpha x_{1}\right)^{K+1}\left(x_{2}-\rho\right)+\rho\left(\alpha x_{2}\right)^{K+1}\left(x_{1}-\rho\right)\right)}$. Now

$$
q_{0}^{\bar{r} \lambda}(0, z)=\frac{R_{K}}{1-z}\left[R_{K+1}^{-1}-z \rho^{K} A_{0}^{\bar{r} \lambda}(0, z)\right],
$$

where $A_{0}^{\bar{r} \lambda}(0, z)=\frac{\left(1-\alpha x_{1}\right) \alpha x_{1} \frac{\left(1-x_{1}^{K}\right)}{\left(1-x_{1}\right)}}{z^{2} \alpha^{2}\left(\rho\left(\alpha x_{1}\right)^{K+1}\left(x_{2}-\rho\right)+\rho\left(\alpha x_{2}\right)^{K+1}\left(x_{1}-\rho\right)\right)}$. Thus,

$$
\begin{aligned}
q_{0}^{\bar{r} \lambda}(0, \bar{r} z) & =\frac{R_{K}}{1-\bar{r} z}\left[R_{K+1}^{-1}-\bar{r} z \rho^{K} A_{0}^{\bar{r} \lambda}(0, \bar{r} z)\right] \\
& =\frac{R_{K}}{1-\bar{r} z}\left[R_{K+1}^{-1}-z \rho^{K} A_{r}^{\lambda}(0, z)\right] .
\end{aligned}
$$

Hence (i) follows. Now,

$$
P_{r}^{\lambda}(0, n)=\left.\frac{1}{(n-1) !} \frac{\partial^{n-1} q_{r}^{\lambda}(0, z)}{\partial z^{n-1}}\right|_{z=0}=\left.\frac{1}{(n-1) !} \bar{r}^{n} \frac{\partial^{n-1} q_{0}^{\bar{r} \lambda}(0, \bar{r} z)}{\partial(\bar{r} z)^{n-1}}\right|_{z=0}
$$


from which (ii) follows.

Proposition 1 yields the following Corollary:

Corollary 2 The probability of losing one packet out of $n$ consecutive packets, i.e., $P(1, n)$ is given by

$$
P(1, n)=\left.\left[z^{n-1}\right] \frac{\partial q(y, z)}{\partial y}\right|_{y=0}=\left[z^{n-1}\right] F_{1}(z)+\left[z^{n-1}\right] F_{2}(z)
$$

with

$$
\begin{gathered}
F_{1}(z)=\frac{R_{K}}{1-\bar{r} z} \bar{r}\left[R_{K-1}^{-1}-z(\alpha \rho)^{K+1} A(0, z)\right]\left(-1+\frac{z r \rho}{1-\bar{r} z}\right), \\
F_{2}(y)=\frac{R_{K}}{1-\bar{r} z}\left[R_{K-1}^{-1}+\rho^{K}-z(\alpha \rho)^{K+1} \bar{r} \dot{A}(0, z)+r z(\alpha \rho)^{K} B(0, z)\right],
\end{gathered}
$$

where $A(0, z)$ and $B(0, z)$ are values at $y=0$ of $A(y, z)$ and $B(y, z)$ defined in Proposition 1 and $\dot{A}(0, z)$ is the derivative of $A(y, z)$ with respect to $y$, evaluated at $y=0$.

One can derive expressions for $A(0, z), B(0, z), \dot{A}(0, z)$ from 8 and hence an explicit expression for $P(1, n)$ by looking for the corresponding coefficients in the Taylor's expansions of $F_{1}$ and $F_{2}$. But finding Taylor's expansions may be computationally involved. In Sec. 5 we provide an alternative approach for directly evaluating $P_{\rho}(>j, n), \forall j, 0 \leq j \leq n-1$. Of course for $j=n, P_{\rho}(>n, n)=0$.

\section{Numerical Examples}

In this section we compare the loss probabilities of a whole group of $n$ consecutive packets, which we call a block, with and without $j$ additional redundant packets. The group of packets that include the original block plus the additional redundant packets (if these are added) is called a frame. If at least $n$ packets out of these consecutive $n+j$ packets reach the destination then no loss of frame occurs. In this section we restrict ourselves to the case of $j=0$, i.e., no redundancy and $j=1$, one redundant packet per $n$ packets. Without loss of generality, we may scale the time so that the service rate is unity: $\mu=1$. In the numerical examples we are looking only at the random losses in the incoming link with probability $r$ and congestion losses. We take $K=25$. When we numerically compared $P_{\rho}(>0, n)$ with $P_{\rho}(>1, n+1)$ we always obtained $P_{\rho}(>1, n+1)<P_{\rho}(>0, n)$, which should be of no surprise: this observation means that if redundancy is added in such a way that the total load on the system remains unchanged then indeed redundancy improves performance in terms of loss probabilities. However, the assumption that the total load remains the same means that the throughput of the useful information decreases (in real time applications this would mean that a higher compression rate should be used before transmission). This type of comparison 


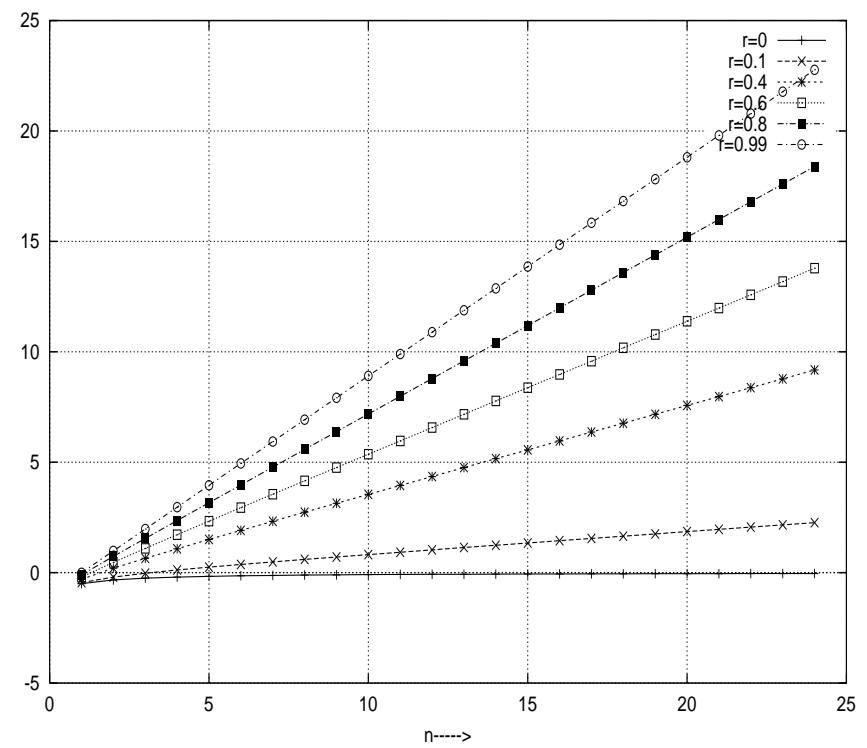

Figure 2: $\frac{\frac{n}{n+1} P(\leq 1, n+1)-P(0, n)}{P(0, n)}$ as a function of $n$ for varying $r$ with $\lambda=0.3$

(keeping the total load unchanged) has not been performed previously in $[7,5,4,9]$ even for the case of congestion losses only. E.g., if we add $k$ redundant packets to $n$ (which gives frames of $n+k$ ) and if the load is unchanged, then this means that the throughput of useful information carried by a frame has decreased by a factor of $n /(n+k)$. Yet we have less losses of packets. Thus the question that needs to be addressed is whether we gain in goodput in this case. Let us define the goodput as the throughput arriving well to the destination. Then this is given by

$$
\text { (input rate of blocks) } \times n /(n+k) \times P_{\rho}(\leq k, n+k) \text {. }
$$

So a meaningful thing to compare is $P_{\rho}(0, n)$ with $\frac{n}{n+1} P_{\rho}(\leq 1, n+1)$ for fixed $\lambda$. In Fig. 2, we plot the relative gain, i.e.,

$$
\frac{\frac{n}{n+1} P(\leq 1, n+1)-P(0, n)}{P(0, n)} .
$$

From Fig. (2) we observe that the benefits of adding FEC grows as the amount of random losses increases, and also as $n$ increases. Also for very low $r$ (very close to 0 ) and very low $n$ (as compared to buffer size) we loose by adding FEC. Fig. (3) plots the same curve for $\lambda=0.99$. We observe that curves for $\lambda=0.3$ and $\lambda=0.99$ are identical $r \geq 0.1$ and larger $n$ and for $r$ close to 0 the difference is very small.

Remark 2 Consider a scenario in which there are only random losses (with probability $r$ ) and no congestion losses. Then we have:

$$
P_{\rho}(0, n)=(1-r)^{n}, \quad P_{\rho}(1, n)=n r(1-r)^{n-1} .
$$




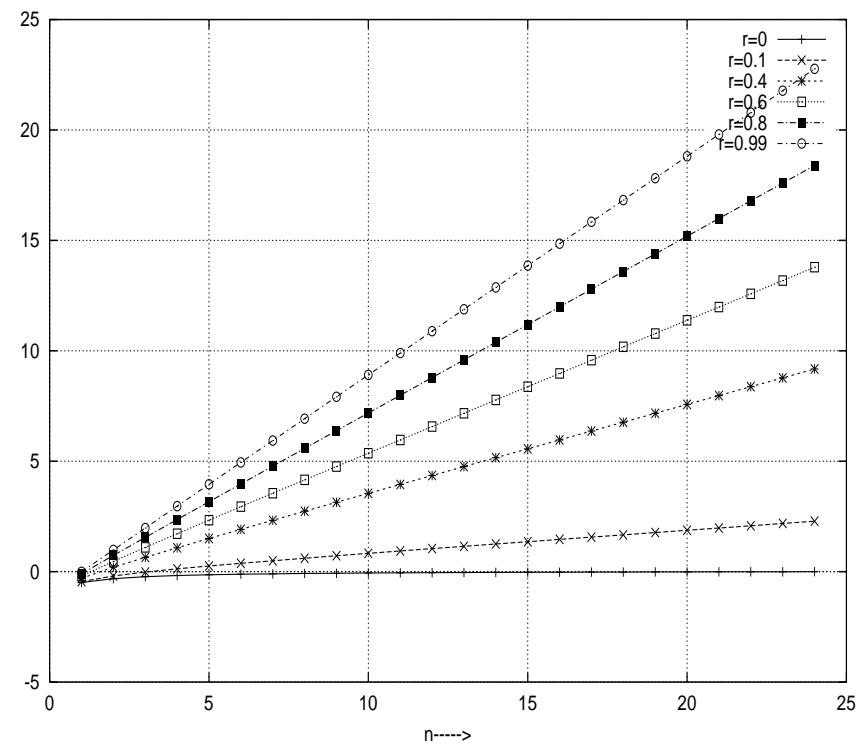

Figure 3: $\frac{\frac{n}{n+1} P(\leq 1, n+1)-P(0, n)}{P(0, n)}$ as a function of $n$ for varying $r$ with $\lambda=0.99$

If we want to study the effect of adding FEC on recovering from different type of losses we can compare the relative gain defined in (11) for the cases when $r=0$ (congestion losses but no random losses) to the case when there are no congestion losses but only random losses with loss probabilities given by (12). We plot this comparison in Fig. (4) and observe that FEC is more helpful in recovering from random losses than congestion losses.

Next we look at the case where the transmission of useful information is kept unchanged when adding redundancy. This implies that the total packet arrival rate increases due to adding redundancy. We assume that the rate at which frames arrive is the same for the two cases and is given by $x$. In the case of no redundancy, the rate at which packets arrive is $\lambda=\rho=n x$ and in case of redundancy $\lambda=\rho=(n+1) x$. A frame is lost in the latter case if more than one packet is lost out of $n+1$ consecutive packets. We are thus interested in the difference $D=P_{n x}(>0, n)-P_{(n+1) x}(>1, n+1)$. If $D>0$ then the redundancy decreases the loss probability of messages. Observe that

$$
\begin{aligned}
D & =1-P_{n x}(0, n)-\left[1-P_{(n+1) x}(0, n+1)-P_{(n+1) x}(1, n+1)\right] \\
& =P_{(n+1) x}(1, n+1)+P_{(n+1) x}(0, n+1)-P_{n x}(0, n) .
\end{aligned}
$$

We next plot the relative gain $\frac{D}{P_{n x}(>0, n)}$ as a function of $n$ for $x=0.03$ (this means the load $n x$, varies from 0.03 (for $n=1$ ) to 0.75 (for $n=25$ )) in Fig. 5 and for $x=0.4$ (load varying from 0.4 to 10) in Fig. 6. The curves show that for fixed $r$, there exists a value of the frame size at which the gain obtained by adding FEC as defined in (13) is maximum. These figures can thus be used in order to optimize the size of blocks to which we should add FEC. 


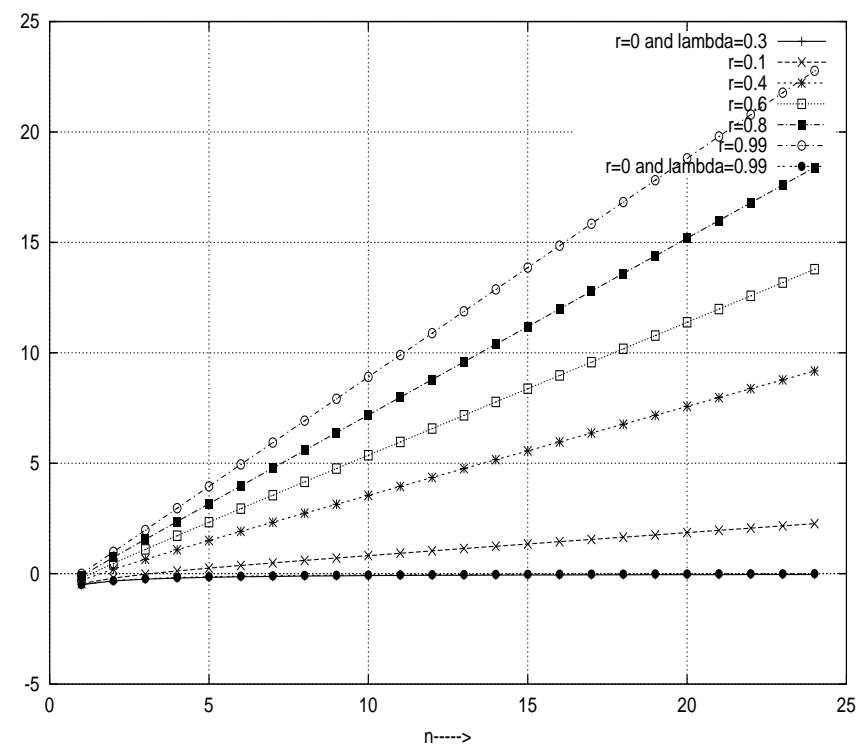

Figure 4: Gain $\frac{\frac{n}{n+1} P(\leq 1, n+1)-P(0, n)}{P(0, n)}$ as a function of $n$ for $r$ varying from 0.1 to 0.99 for the scenario when there are no congestion losses. Also shown is the gain when there are no random losses $(r=0)$ and only congestion losses with $\lambda=0.3$ and $\lambda=0.99$. Observe that the curves for $r=0$ and $\lambda=0.3$ and $\lambda=0.99$ have negligible differences.

Remark 3 From Fig. 5 we observe that for $r=0.1$, adding one redundant packet for a block size of 10 packets will result in the maximum gain in $D$. The redundant packet can be constructed as follows: Let the packet sizes be say $M$ bits. Then the $i t h, 1 \leq i \leq M$, bit of the redundant packet is obtained by an $X 0 R$ operation on the ith bits of all the 10 packets.

All the above curves establish that we benefit from adding redundancy when $r$ is not very small, and this is a valid remark or observation at any load. However when the random loss probability is very low (close to 0 ) we may loose by adding redundancy.

\section{Combinatorial Approach Using Ballot Theorems}

We next employ combinatorial arguments together with the Ballot theorems [6] to alternatively obtain explicit expressions for all the probabilities of the previous section. In particular, we shall find the probability $P_{i}^{a}(j, n)$. Let us denote the loss probabilities in a system with no random losses but only congestion losses and Poisson arrival process with parameter $\bar{r} \lambda$ by $\bar{P}_{i}^{a}(j, n), 0 \leq j \leq n$. Observe that these probabilities can be obtained from [9]. 


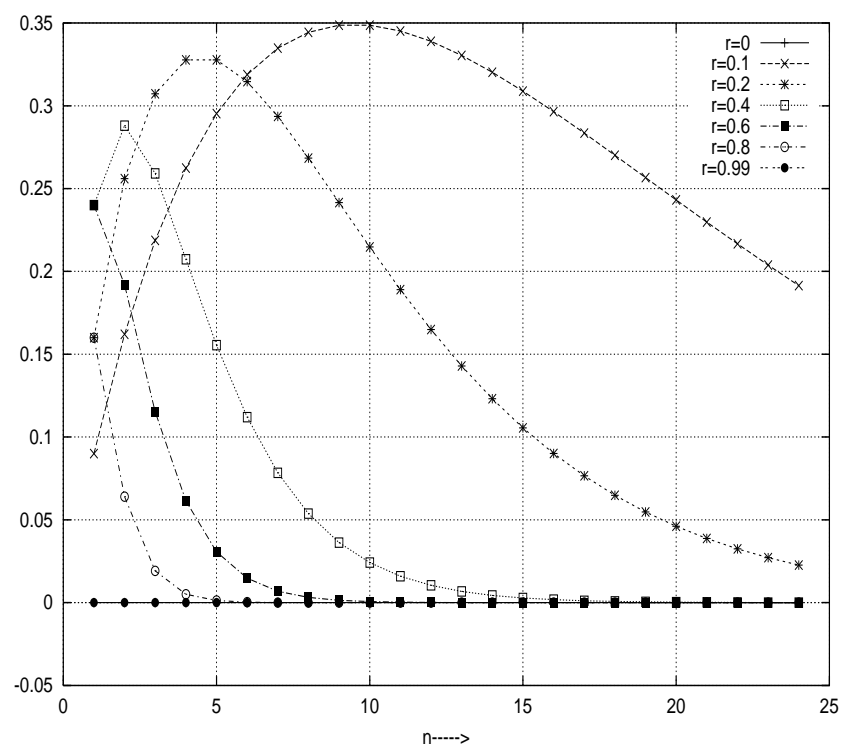

Figure 5: $\frac{D}{P_{n x}(>0, n)}$ as a function of $n$ for different $r$ and $x=0.03$. Observe that the load changes with $n$ also.

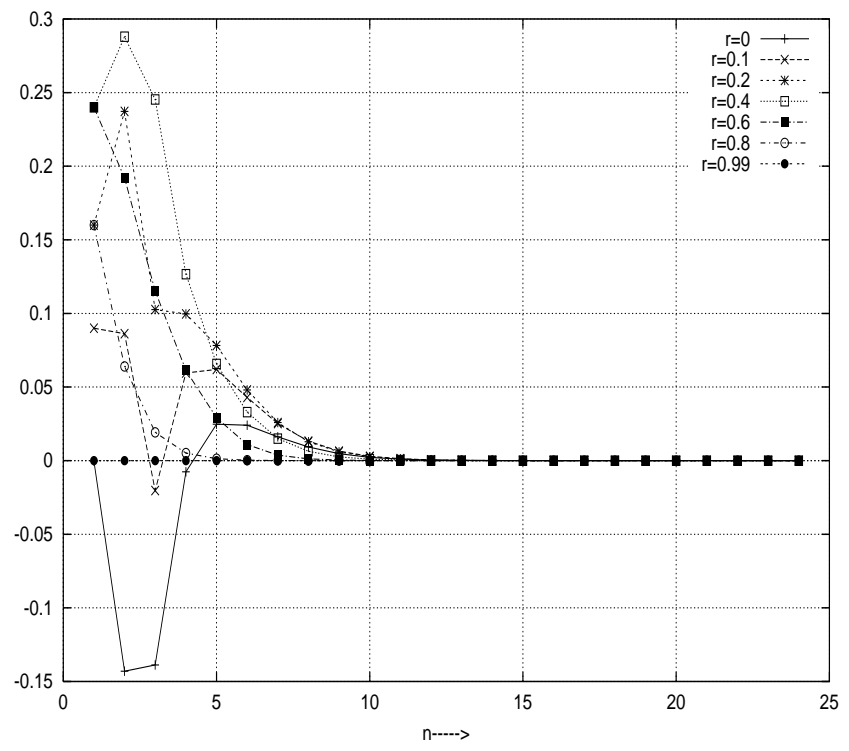

Figure 6: $\frac{D}{P_{n x}(>0, n)}$ as a function of $n$ for different $r$ and $x=0.4$ 
Consider the case when $j^{2}$ losses consist of $j_{r}\left(0 \leq j_{r} \leq j\right)$ random losses and $j_{c}\left(0 \leq j_{c} \leq j\right)$ congestion losses. For $n=1$ we have, $P_{i}^{a}(j, n)$ from (4) and (5). We shall now deal with $n \geq 2$. For $j_{r}=0$, we have $P_{i}^{a}(j, n)=(1-r)^{n} \bar{P}_{i}^{a}\left(j_{c}, n\right)$ with $j_{c}=j$ and $\bar{P}_{i}^{a}\left(j_{c}, n\right)$ given from [9]. We next consider the case for $j_{r} \geq 1$. The number of ways in which $j_{r}$ random losses can occur among $j$ losses is $\left(\begin{array}{l}j \\ j_{c}\end{array}\right)$. We calculate the probability of one such outcome. The probability depends on the position of the lost packets in the frame. Let us denote by $r_{i}$ the position of the $i$ th random loss, $1 \leq i \leq j_{r}$ in the original frame. Also $i \leq r_{i} \leq n-\left(j_{r}-i\right)$. Thus $r_{1}=1$, when the first packet was lost by random loss and $r_{j_{r}}=n$, when the last packet was lost by random loss.

The following analysis is for the case of $j_{r} \geq 2, r_{1} \neq 1, r_{j_{r}} \neq n$ and $j_{r}=1, r_{1} \neq 1$ or $n$. We shall supplement the analysis with the other cases at appropriate places. Observe that the random losses can be isolated or they can occur in burst. In fact since our message length is finite $(n)$, the probability that all the random losses occur in a burst is $>0{ }^{3}$. Also observe that only the packets of the original message which are not subject to random losses have the potentials of getting lost at the queue due to congestion (as we have assumed these are the only packets that actually reach the queue). Thus we shall look at the packets of the original message between consecutive random loss events. A random loss event is formed of consecutive random losses. Say that consecutive packets actually coming to the queue and are not corrupted due to link losses form an interval. Let $T$ be the number of such intervals. Thus $T$ includes:

- The interval consisting of packets coming to the queue before the first random loss event (if $r_{1} \neq 1$ ).

- The interval consisting of packets coming to the queue after the last random loss event (if $r_{j_{r}} \neq n$ ).

- The interval consisting of packets coming to the queue between two random loss events.

Let $k_{i}$ be the number of consecutive random losses in the $i$ th random loss event.

Remark 4 The value of $T$ depends on $n, j_{r}$ and the position of random losses. For e.g., for $n=j_{r}, T=0$, for $n=j_{r}+1, T=1$ etc.

Define

$$
z(t):=\sum_{h=1}^{t} k_{h} .
$$

\footnotetext{
${ }^{2}$ Observe that here we are looking at the case when the random losses (if any) occur before the frame enters the buffer. The complementary case of random losses occurring after the frame leaves the node can be handled as discussed in Remark 1. And then one can obtain the loss probabilities for the case when random losses can occur both in the outgoing and in the incoming link.

${ }^{3}$ Although bursty loss occurrence is more a characteristic of congestion losses.
} 
We now distribute the $j_{c}$ congestion losses in the $T$ intervals of lengths $r_{1}-1, r_{1+k_{1}}-r_{k_{1}}-$ $1, r_{1+k_{1}+k_{2}}-r_{k_{1}+k_{2}}-1, \ldots, n-r_{z(T-1)}-1$. Let $n_{y}$ be the number of congestion losses in the $y$ th such interval. Observe that (for $2 \leq y \leq T-1$ )

$$
0 \leq n_{y} \leq \min \left(r_{1+z(y-1)}-r_{z(y-1)}-1, j_{c}\right) .
$$

For $y=1,0 \leq n_{y} \leq \min \left(r_{1}-1, j_{c}\right)$ and for $y=T, 0 \leq n_{y} \leq \min \left(n-r_{z(T-1)}-1, j_{c}\right)$. Also, $n_{y}$ satisfy $\sum_{y=1}^{T} n_{y}=j_{c}$. Now the number of ways in which $n_{y}$ losses can occur in the $y$ th interval is

$$
\left(\begin{array}{c}
r_{1+z(y-1)}-r_{z(y-1)}-1 \\
n_{y}
\end{array}\right)
$$

for $2 \leq y \leq T-1$ and is $\left(\begin{array}{l}r_{1}-1 \\ n_{1}\end{array}\right)$ for $y=1,\left(\begin{array}{l}n-r_{z(T-1)}-1 \\ n_{T}\end{array}\right)$ for $y=T$. We shall calculate the probability of one such event. We shall look at three types of intervals: $A$-starts with the first arrival after a random loss event and ends with the last arrival before a random loss event; $B$-starts with the arrival of the first packet of the message (if $r_{1} \neq 1$ ) and ends with the last arrival before the first random loss event; $C$-starts with the first arrival after the last random loss event and ends with the arrival of the last packet of the message (if $r_{j_{r}} \neq n$ ).

In a sample path with $j_{r} \geq 2, r_{1} \neq 1, r_{j_{r}} \neq n$, and with $A_{i}$ an interval of type $A$, the order of occurrence of the intervals is $B \rightarrow A_{1} \rightarrow A_{2} \ldots \rightarrow A_{T-2} \rightarrow C$. For $j_{r} \geq 2, r_{1}=1, r_{j_{r}} \neq n$, the order is $A_{1} \rightarrow A_{2} \ldots \rightarrow A_{T-1} \rightarrow C$ and no interval of type $B$. For $j_{r} \geq 2, r_{1} \neq 1, r_{j_{r}}=n$, the order is $B \rightarrow A_{1} \ldots A_{T-1}$ and no interval of type $C$. Similarly, for $j_{r} \geq 2, r_{1}=1, r_{j_{r}}=n$, there will be no interval of type either $B$ or of type $C$. For $j_{r}=1$, there can either be intervals $B \rightarrow C$ or $C$ or $B$ and no interval of type $A$ can occur.

Let the queue length at the beginning of the $y$ th interval be $\alpha$ and at the end of the interval be $\beta$.

We shall first calculate the probability of a path that starts with $\alpha$ packets in the buffer, ends with $\beta$ packets in the buffer, has $n_{y}$ losses in it by congestion and consists of $a_{y}=$ $\left(r_{1+z(y-1)}-r_{z(y-1)}-1\right)$ arrival events. We employ the arguments as in [9] to evaluate this probability. However here in our analysis we also need to know the queue length at the arrival of the last packet of an interval. We shall denote this probability by $P_{(\alpha, \beta)}\left(n_{y}, a_{y}\right)$. Let $f_{j}$ denote the $j$ th lost packet. We shall decompose an interval into three types of events as follows: (i) $\mathcal{V}_{\alpha}\left(f_{1}\right)$-the first packet to be lost is $f_{1}$ given that upon the arrival of the first packet of the interval there are $\alpha$ packets in the buffer; (ii) $\mathcal{S}\left(f_{l}, f_{l+1}\right)$-packet $f_{l+1}$ is lost given that packet $f_{l}$ was lost; (iii) $\mathcal{U}\left(f_{n_{y}}, \beta\right)$-packet $f_{n_{y}}$ is the last to be lost and the queue length at the arrival of the last packet of the interval is $\beta$.

Observe that an interval consists of the succession of events $\mathcal{V}_{\alpha}\left(f_{1}\right), \mathcal{S}\left(f_{1}, f_{2}\right), \mathcal{S}\left(f_{2}, f_{3}\right)$, $\ldots, \mathcal{S}\left(f_{n_{y}-1}, f_{n_{y}}\right), \mathcal{U}\left(f_{n_{y}}, \beta\right)$. Let $v_{\alpha}\left(f_{1}\right), s\left(f_{l}, f_{l+1}\right)$ and $u\left(f_{n_{y}}, \beta\right)$ be the probabilities of the event $\mathcal{V}_{\alpha}\left(f_{1}\right), \mathcal{S}\left(f_{l}, f_{l+1}\right)$ and $\mathcal{U}\left(f_{n_{y}}, \beta\right)$, respectively. Thus

$$
P_{(\alpha, \beta)}\left(n_{y}, a_{y}\right)=\sum_{f_{1}=1}^{a_{y}-n_{y}+1} \sum_{f_{2}=f_{1}+1}^{a_{y}-n_{y}+2} \ldots \sum_{f_{n_{y}}=f_{n_{y}-1}+1}^{a_{y}} v_{\alpha}\left(f_{1}\right) s\left(f_{1}, f_{2}\right) \ldots s\left(f_{n_{y}-1}, n_{y}\right) u\left(f_{n_{y}}, \beta\right) .
$$


The computation of the probabilities $v_{\alpha}\left(f_{1}\right)$ and $s\left(f_{l}, f_{l+1}\right)$ is similar to that in [9]. However the computation of $u\left(f_{n_{y}}, \beta\right)$ requires some combinatorial arguments. We shall, for completeness summarize the results in the following Proposition and shall provide the proof for the expression for $u\left(f_{n_{y}}, \beta\right)$.

Proposition 2 The probabilities $v_{\alpha}\left(f_{1}\right), s\left(f_{l}, f_{l+1}\right)$ and $u\left(f_{n_{y}}, \beta\right)$ are given as

$$
\begin{aligned}
& v_{K}\left(f_{1}\right)=\left\{\begin{array}{cc}
1 & f_{1}=1 \\
0 & \text { o.w. }
\end{array} ; \quad v_{\alpha}\left(f_{1}\right)=\left\{\begin{array}{cc}
0 & f_{1} \leq K-\alpha \\
\frac{\rho}{\rho+1} . \phi_{2 f_{1}-K+\alpha-3}(\alpha+1, K) & \text { o.w. }
\end{array} \neq K\right.\right. \\
& s\left(f_{l}, f_{l+1}\right)=\frac{\rho}{\rho+1} \cdot \phi_{2\left(f_{l+1}-f_{l}-1\right)}(K, K) \\
& u\left(f_{n_{y}}, \beta\right)=\left\{\begin{array}{cc}
\phi_{2\left(a_{y}-f_{n_{y}}\right)+K-\beta}(K, \beta) & f_{n_{y}}<a_{y} \\
1 & f_{n_{y}}=a_{y} \text { and } \beta=K \\
0 & f_{n_{y}}=a_{y} \text { and } \beta \neq K
\end{array}\right.
\end{aligned}
$$

where $\phi_{\eta}(\alpha, \beta)$ is defined as the probability of a path that starts with $\alpha$ packets in the buffer, ends with $\beta$ packets in the buffer and consists of $\eta$ events (arrivals and departures) and is defined as

$$
\phi_{\eta}(\alpha, \beta)=\epsilon_{\eta}(\alpha, \beta)+\sum_{r=1}^{\mathcal{H}} W_{\alpha} Y^{r-1} Z^{\mathcal{T}}
$$

where, for $\alpha \geq 1, \beta \geq 1$

$$
\begin{aligned}
\epsilon_{\eta}(\alpha, \beta)= & \\
& \sum_{\Upsilon}\left[\left(\begin{array}{c}
\eta \\
\frac{\eta+\alpha-\beta}{2}-\Upsilon(K+1)
\end{array}\right)-\left(\begin{array}{c}
\eta \\
\frac{\eta-\alpha-\beta}{2}-\Upsilon(K+1)
\end{array}\right)\right]\left(\frac{\rho}{1+\rho}\right)^{\frac{\eta-\alpha+\beta}{2}}\left(\frac{1}{1+\rho}\right)^{\frac{\eta+\alpha-\beta}{2}}, \\
W_{\alpha}= & \left(\epsilon_{\alpha}(\alpha, 0), \epsilon_{\alpha+2}(\alpha, 0), \ldots, \epsilon_{\alpha+2(\mathcal{H}-1)}(\alpha, 0)\right) \\
Z= & \left(\epsilon_{\eta-\alpha}(0, \beta), \epsilon_{\eta-\alpha-2}(0, \beta), \ldots, \epsilon_{\eta-\alpha-2(\mathcal{H}-1)}(0, \beta)\right) \\
Y= & \left(\begin{array}{ccccc}
0 & \epsilon_{2}(0,0) & \epsilon_{4}(0,0) & \ldots & \epsilon_{2(\mathcal{H}-1)}(0,0) \\
0 & 0 & \epsilon_{2}(0,0) & \ldots & \epsilon_{2(\mathcal{H}-2)}(0,0) \\
\vdots & \vdots & \vdots & \vdots & \vdots \\
0 & 0 & 0 & \ldots & \epsilon_{2}(0,0) \\
0 & 0 & 0 & \ldots & 0
\end{array}\right) \\
\mathcal{H}= & 1+\frac{\eta-\alpha-\beta}{2}
\end{aligned}
$$


and $\epsilon_{\eta}(0, \beta)=\epsilon_{\eta-1}(1, \beta), \beta \geq 1, \epsilon_{\eta}(\alpha, 0)=\frac{1}{1+\rho} \epsilon_{\eta-1}(\alpha, 1), \alpha \geq 1, \epsilon_{\eta}(0,0)=\frac{1}{1+\rho} \epsilon_{\eta-2}(1,1)$ where $-\infty<\Upsilon<\infty$ takes on values in the sum in the definition of $\epsilon_{\eta}(\alpha, \beta)$ in (18) so that the binomial coefficients are proper, for e.g. in the first sum in (18) $\frac{\eta+\alpha-\beta}{2}>\Upsilon(K+1)$ and $\eta>\frac{\eta+\alpha-\beta}{2}-\Upsilon(K+1)$.

Proof: For proofs of Eqs. (14) and (15) see [9]. We shall here provide a proof for Eq. (16). Observe that for $f_{n_{y}}=a_{y}, \beta=K, u\left(f_{n_{y}}, \beta\right)=1$. For $\beta \neq K, u\left(f_{n_{y}}, \beta\right)=0$. We look at the case $f_{n_{y}}<a_{y}$. Observe that after the $f_{n_{y}}$ th packet there are $a_{y}-f_{n_{y}}$ more packets to come. And at the loss of $f_{n_{y}}$ th packet, the buffer is full, that is queue length is $K$. Thus we need the probability of a path that starts when there are $K$ packets in the buffer ends with $\beta$ packets, consists of $2\left(a_{y}-f_{n_{y}}\right)+K-\beta$ events (arrivals and service completions) and no packets are lost. This is nothing but the probability $\phi_{2\left(a_{y}-f_{n_{y}}\right)+K-\beta}(K, \beta)$ from the definition in (17).

We also need the probability of the evolution of a path after the end of interval $A_{i}$ and before the start of interval $A_{i+1}$ and having $k_{i}(\geq 1)$ packets lost by random losses. Observe that the duration of this random loss event has the distribution of the sum of $k_{i}+1$ independent $\exp (\lambda)$ distributed random variables, i.e., $\operatorname{Erlang}\left(k_{i}+1, \lambda\right)$. Let $X_{i}$ be the number of service completions $\exp (\mu)$ in an interval with distribution $F * F * \ldots(k-$ times $)=F^{* k}$ where $F \sim \exp (\lambda)$ and $*$ denotes the convolution operation. Then the probability that $A_{i}$ ends with $\beta_{1}$ packets (including the last arrival in the interval $A_{i}$ ) in the buffer and $A_{i+1}$ starts with $\beta_{2}$ packets (not including the first arrival in the interval $A_{i+1}$ ) in the buffer and has $k_{i}$ random losses can be written as

$$
P\left(X_{i}=\beta_{1}-\beta_{2}, k_{i}\right)=\left\{\begin{array}{cc}
\int_{0}^{\infty} \frac{e^{-\mu s}(\mu s)^{\left(\beta_{1}-\beta_{2}\right)}}{\left(\beta_{1}-\beta_{2}\right) !} d F^{*\left(k_{i}+1\right)}(s) & \text { if } 0<\beta_{2} \leq \beta_{1} \\
\sum_{m=\beta_{1}}^{\infty} \int_{0}^{\infty} \frac{e^{-\mu s}(\mu s)^{m}}{m !} d F^{*\left(k_{i}+1\right)}(s) & \text { if } \beta_{2}=0 \\
0 & \beta_{2}>\beta_{1} .
\end{array}\right.
$$

Remark 5 Indeed, the end of service times are a Poisson process with intensity $\mu$. The $P G F$ of the number of such points during a fix interval $T$ is $G(z)=\exp (-\mu(1-z) T)$. If $T$ is a random interval then it is $G(z)=E\left[\exp (-\mu(1-z) T]=T^{*}\left(\mu(1-z)\right.\right.$ where $T^{*}(s)$ is the Laplace Stieltjes transform of $T$. If $T$ were exponential $(\lambda)$ then this would give

$$
G(z)=\frac{\lambda}{\lambda+\mu(1-z)}=\frac{1}{z} \frac{\theta z}{1-(1-p) z} \text { where } \theta=\frac{\lambda}{\lambda+\mu}=\frac{\rho}{1-\rho} .
$$

We see that $G(z)$ is the $P G F$ of $Y=X-1$ where $X$ has a geometric distribution with parameter $\theta$, so $P(Y=n)=(1-\theta)^{n} \theta$. The number of points in an Erlang $\left(k_{i}+1, \lambda\right) R V$, 
say $X_{i}$, has thus the distribution of the convolution of $k_{i}+1$ copies of $Y$, which gives:

$$
P\left(X_{i}=n\right)=\sum_{y_{1}+\ldots+y_{n}=k_{i}+1} \frac{\left(k_{i}+1\right) !}{y_{1} ! y_{2} ! \ldots y_{n} !} \theta^{n}(1-\theta)^{k_{i}+1} .
$$

This can now be used to for the expressions in (19).

We will now consider a path in which the first packet (out of $n$ packets in a frame) sees $i$ packets in the buffer, and out of $n$ packets in a frame, $j_{r}$ packets are lost by random losses $j_{c}$ packets are lost by congestion losses, $j_{c}+j_{r}=j$ with $T$ intervals. Let $r_{i}$ be the position of the $i$ th random loss. Let $P_{p}^{i}\left(j_{c}, j_{r}, T, n\right)$ be the probability of such a path ${ }^{4}$. Then for $r_{1} \neq 1$ and $r_{j_{r}} \neq n, j_{r} \geq 2$ and for $j_{r}=1$ and $r_{1} \neq 1$ or $n$ :

$$
\begin{aligned}
& P_{p}^{i}\left(j_{c}, j_{r}, T, n\right) \\
& =r^{j_{r}}(1-r)^{n-j_{c}} \sum_{\beta_{g}=0,0 \leq g \leq T-1}^{\beta_{g}=K} \sum_{\alpha_{h}=1,0 \leq h \leq T-1}^{\alpha_{h}=K} \sum_{r_{1}=2}^{n-j_{r}} \sum_{k_{1}=1}^{j_{r}} \sum_{k_{2}=1}^{j_{r}-k_{1}} \ldots \sum_{k_{T-2}=1}^{j_{r}-\sum_{h=1}^{T-3} k_{h}}
\end{aligned}
$$

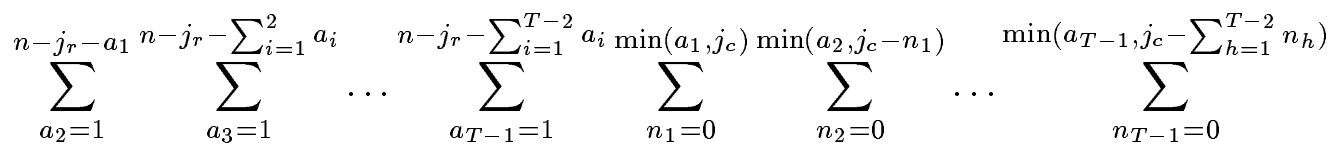

$$
\begin{aligned}
& C\left(n_{1}, a_{1}\right) P_{\left(i, \beta_{0}\right)}\left(n_{1}, a_{1}\right) P\left(X_{1}=\beta_{0}-\alpha_{1}, k_{1}\right) C\left(n_{2}, a_{2}\right) P_{\left(\alpha_{1}, \beta_{1}\right)}\left(n_{2}, a_{2}\right) \\
& P\left(X_{2}=\beta_{1}-\alpha_{2}, k_{2}\right) \ldots C\left(n_{T-1}, a_{T-1}\right) P_{\left(\alpha_{T-2}, \beta_{T-2}\right)}\left(n_{T-1}, a_{T-1}\right) \\
& P\left(X_{T-1}=\beta_{T-2}-\alpha_{T-1}, k_{T-1}\right) C\left(n_{T}, a_{T}\right) P_{\left(\alpha_{T-1}, \beta_{T-1}\right)}\left(n_{T}, a_{T}\right) .
\end{aligned}
$$

where $\sum_{k=1}^{i} f_{k}=0$ for $i \leq 0$ and $a_{1}=r_{1}-1, a_{T}=n-j_{r}-\sum_{i=1}^{T-1} a_{i}, k_{T-1}=j_{r}-\sum_{h=1}^{T-2} k_{h}$, $n_{T}=j_{c}-\sum_{h=1}^{T-1} n_{h}$. Also $C(n, a)=\frac{a !}{(a-n) ! n !}$. We now consider the other cases:

- $j_{r}=1, r_{1}=1$ : Here $T=1$ and $k_{1}=1$. For this case we can write $P_{p}^{i}\left(j_{c}, j_{r}, T, n\right)$ as $r(1-r)^{n-1} \sum_{\beta_{0}=0}^{i} P\left(X_{0}=i-\beta_{0}, 1\right) \bar{P}_{\beta_{0}}^{a}\left(j_{c}, n-1\right)$ with $\bar{P}_{\beta_{0}}^{a}(.,$.$) obtained as [9] and$ $P\left(X_{0}=.,.\right)$ having same distribution as $(19)$.

- $j_{r}=1, r_{1}=n$ : Here again $T=1$ and $k_{1}=1$. We can write $P_{p}^{i}\left(j_{c}, j_{r}, T, n\right)$ as $r(1-r)^{n-1} \bar{P}_{i}^{a}\left(j_{c}, n-1\right)$ with $\bar{P}_{i}^{a}(.,$.$) obtained as in [9].$

- $j_{r} \geq 2, r_{1}=1, r_{j_{r}} \neq n$ : We have $P_{p}^{i}\left(j_{c}, j_{r}, T, n\right)=$

$$
r^{j_{r}}(1-r)^{n-j_{r}} \sum_{k_{1}=1}^{j_{r}} \sum_{\beta_{0}=0}^{i} P\left(X_{0}=i-\beta_{0}, k_{1}\right) P_{p}^{\beta_{0}}\left(j_{c}, j_{r}-k_{1}, T, n-k_{1}\right) .
$$

with $P_{p}^{\beta_{0}}(., ., .,$.$) given as in (20)$.

\footnotetext{
${ }^{4}$ We use the subscript $p$ to distinguish the notation from Section 3
} 
- $j_{r} \geq 2, r_{1} \neq 1, r_{j_{r}}=n$ : We have $P_{p}^{i}\left(j_{c}, j_{r}, T, n\right)$ :

$$
\begin{aligned}
= & r^{j_{r}}(1-r)^{n-j_{c}} \sum_{\beta_{g}=0,0 \leq g \leq T-1}^{\beta_{g}=K} \sum_{\alpha_{h}=1,0 \leq h \leq T-1}^{\alpha_{h}=K} \sum_{r_{1}=2}^{n-j_{r}} \sum_{k_{T}=1}^{j_{r}} \sum_{k_{T-1}=1}^{j_{r}-k_{T}} \ldots \sum_{k_{2}=1}^{j_{r}-\sum_{h=3}^{T} k_{h}} \\
& \sum_{a_{2}=1}^{n-j_{r}-a_{1}} \sum_{a_{3}=1}^{n-j_{r}-\sum_{i=1}^{2} a_{i}} \ldots \sum_{a_{T-1}=1}^{n-j_{r}-\sum_{i=1}^{T-2}} \sum_{n_{1}=0} \sum_{n_{2}=0}^{\min \left(a_{1}, j_{c}\right)} \sum_{\substack{\min \left(a_{2}, j_{c}-n_{1}\right) \\
\min \left(a_{T-1}, j_{c}-\sum_{h=1}^{T-2} n_{h}\right)}} \sum_{n_{T-1}=0} \\
& C\left(n_{1}, a_{1}\right) P_{\left(i, \beta_{0}\right)}\left(n_{1}, a_{1}\right) P\left(X_{1}=\beta_{0}-\alpha_{1}, k_{1}\right) C\left(n_{2}, a_{2}\right) P_{\left(\alpha_{1}, \beta_{1}\right)}\left(n_{2}, a_{2}\right) \\
& P\left(X_{2}=\beta_{1}-\alpha_{2}, k_{2}\right) \ldots C\left(n_{T-1}, a_{T-1}\right) P_{\left(\alpha_{T-2}, \beta_{T-2}\right)}\left(n_{T-1}, a_{T-1}\right) \\
& P\left(X_{T-1}=\beta_{T-2}-\alpha_{T-1}, k_{T-1}\right) C\left(n_{T}, a_{T}\right) P_{\left(\alpha_{T-1}, \beta_{T-1}\right)}\left(n_{T}, a_{T}\right) .
\end{aligned}
$$

where $\sum_{k=1}^{i} f_{k}=0$ for $i \leq 0$ and $a_{1}=r_{1}-1, a_{T}=n-j_{r}-\sum_{i=1}^{T-1} a_{i}, k_{1}=j_{r}-\sum_{h=2}^{T} k_{h}$, $n_{T}=j_{c}-\sum_{i=1}^{T-1} n_{h}$.

- $j_{r} \geq 2, r_{1}=1, r_{j_{r}}=n$ : We have $P_{p}^{i}\left(j_{c}, j_{r}, T, n\right)=$

$$
r^{j_{r}}(1-r)^{n-j_{r}} \sum_{k_{1}=1}^{j_{r}} \sum_{\beta_{0}=0}^{i} P\left(X_{0}=i-\beta_{0}, k_{1}\right) P_{p}^{\beta_{0}}\left(j_{c}, j_{r}-k_{1}, T, n-k_{1}\right) .
$$

with $P_{p}^{\beta_{0}}(., ., .,$.$) in last equation given by (21)$.

Having obtained the expressions we have (with appropriate range for values of $T$ )

$$
P_{p}^{i}\left(j_{c}, j_{r}, n\right)=\sum_{T} P_{p}^{i}\left(j_{c}, j_{r}, T, n\right) \quad \text { and } \quad P_{p}^{i}(j, n)=\left(\begin{array}{l}
j \\
j_{c}
\end{array}\right) P_{p}^{i}\left(j_{c}, j_{r}, n\right) .
$$

And finally,

$$
P_{p}(j, n)=\sum_{i=0}^{K} \Pi(i) P_{p}^{i}(j, n)
$$

where $\Pi(i)$ is defined in Equation (1). The probability $P_{p}(j, n)$ here is the same as the probability $P(j, n)$ in Sec. 3 .

\section{Conclusion and Scope of Further Research}

We have studied the steady state loss probabilities of messages in an $M / M / 1 / K$ queue where there are both random losses and congestion losses using an algebraic approach involving generating functions and a second approach based on ballot theorems. The explicit expressions we obtained allowed us to investigate numerically when it is profitable to add FEC, and what 
should the optimal block size be when we add a single redundant packet per block (e.g. using a XOR operation).

Our method can easily be generalized to include multiple sessions (by generalizing the recursions in [4] to include random losses also). Also instead of fixed random loss probabilities, we can include the case where loss probabilities are dependent on the state of the channel, for e.g., one can employ the Gilbert loss model for channels [2] or its generalization ([3]). We can write recursions for the steady state loss probabilities as a function of channel state, say $P_{i}^{a}(j, n, s)$, i.e., conditioned on the state $s$ of the channel upon arrival. If we assume that during the arrival of a message the channel state remains unchanged, say $s$, (this is the case when the time scale of the Markov chain describing the channel is considerably slower than the duration it takes for a message to be served) the unconditional loss probability $P_{i}^{a}(j, s)$ is

$$
P_{i}^{a}(j, n)=\sum_{s=1}^{S} P_{i}^{a}(j, n, s) P_{c}(s)
$$

where $S$ is the total number of possible channel states and $P_{c}(s)$ is the steady state probability that the channel is in state $s$. Another interesting direction will be to model bursty sources. The source can be modeled as an Interrupted Poisson process and again recursive equations can be written for loss probabilities.

\section{Appendix}

Proof of Proposition 1

Define

$$
\pi_{j, n}(x) \triangleq \sum_{i=0}^{K} x^{i} P_{i}^{a}(j, n), \quad n \geq 1, j \geq 0 .
$$

It follows from (6) for $n \geq 2$,

$$
\begin{aligned}
\pi_{j, n}(x)= & \bar{r} \sum_{i=0}^{K-1} x^{i} \sum_{k=0}^{i+1} Q_{i+1}(k) P_{i+1-k}^{a}(j, n-1)+r \sum_{i=0}^{K-1} x^{i} \sum_{k=0}^{i} Q_{i}(k) P_{i-k}^{a}(j-1, n-1) \\
& +x^{K} \sum_{k=0}^{K} Q_{K}(k) P_{K-k}^{a}(j-1, n-1) .
\end{aligned}
$$

We substitute (2) in the last equation, introduce $\pi_{j, n}(x)$ and also use the facts that $\pi_{j, n}(0)=$ $P_{0}^{a}(j, n)$ and $1-\rho \alpha=\alpha$. We then obtain for $n \geq 2, j \geq 1$

$\pi_{j, n}(x)=\bar{r} \sum_{i=0}^{K-1} x^{i}\left(\sum_{k=0}^{i} \rho \alpha^{k+1} P_{i+1-k}^{a}(j, n-1)+\alpha^{i+1} P_{0}^{a}(j, n-1)\right)$ 


$$
\begin{aligned}
& +r \sum_{i=0}^{K-1} x^{i}\left(\sum_{k=0}^{i-1} \rho \alpha^{k+1} P_{i-k}^{a}(j-1, n-1)+\alpha^{i} P_{0}^{a}(j-1, n-1)\right) \\
& +x^{K}\left(\sum_{k=0}^{K-1} \rho \alpha^{k+1} P_{K-k}^{a}(j-1, n-1)+\alpha^{K} P_{0}^{a}(j-1, n-1)\right) \\
= & \bar{r} \sum_{i=0}^{K-1} x^{i}\left(\sum_{k=0}^{i} \rho \alpha^{k+1} P_{i+1-k}^{a}(j, n-1)+\alpha^{i+1} P_{0}^{a}(j, n-1)\right)+r \frac{1-(\alpha x)^{K}}{1-\alpha x} \pi_{j-1, n-1}(0) \\
& +r \frac{\rho \alpha}{1-\alpha x}\left(\pi_{j-1, n-1}(x)-\pi_{j-1, n-1}(0)-x^{K} P_{K}^{a}(j-1, n-1)\right) \\
& -r \frac{\rho \alpha(\alpha x)^{K}}{1-\alpha x}\left(\pi_{j-1, n-1}\left(\alpha^{-1}\right)-\pi_{j-1, n-1}(0)-\alpha^{-K} P_{K}^{a}(j-1, n-1)\right) \\
& +x^{K}\left(\sum_{k=0}^{K-1} \rho \alpha^{k+1} P_{K-k}^{a}(j-1, n-1)+\alpha^{K} P_{0}^{a}(j-1, n-1)\right) \\
= & \frac{\bar{r} \rho \alpha^{2}}{1-\alpha x}\left(\frac{1}{\alpha x} \pi_{j, n-1}(x)-(\alpha x)^{K} \pi_{j, n-1}\left(\alpha^{-1}\right)\right) \\
& -\frac{\bar{r} \rho \alpha^{2}}{1-\alpha x}\left(\frac{1}{\alpha x}-(\alpha x)^{K}\right) \pi_{j, n-1}(0)+\bar{r} \alpha^{1-(\alpha x)^{K}} \\
+ & +\frac{\rho \alpha}{1-\alpha x}\left(\pi_{j-1, n-1}(x)-(\alpha x)^{K} \pi_{j-1, n-1}\left(\alpha^{-1}\right)\right) \\
& +r \alpha \frac{1-(\alpha x)^{K}}{1-\alpha x} \pi_{j-1, n-1}(0)+\alpha \rho(\alpha x)^{K} \pi_{j-1, n-1}\left(\alpha^{-1}\right)+\alpha(\alpha x)^{K} \pi_{j-1, n-1}(0) .
\end{aligned}
$$

Define, with some abuse of notation, the generating function of $P_{i}^{a}(j, n)$

$$
\pi(x, y, z) \triangleq \sum_{j=0}^{\infty} \sum_{n=1}^{\infty} y^{j} z^{n-1} \pi_{j, n}(x) .
$$

When we fix $y$ and $|z|<1$, the above generating function is polynomial in $x$, and therefore an analytic function. In order to use (22), which holds only for $n \geq 2$ and $j \geq 1$, we note that

$$
\begin{aligned}
\sum_{j=1}^{\infty} \sum_{n=2}^{\infty} y^{j} z^{n-1} \pi_{j, n}(x) & =\pi(x, y, z)-\sum_{n=1}^{\infty} z^{n-1} \pi_{0, n}(x)-\sum_{j=0}^{\infty} y^{j} \pi_{j, 1}(x)+\pi_{0,1}(x) \\
& =\pi(x, y, z)-\pi(x, 0, z)-\pi(x, y, 0)+\pi(x, 0,0) .
\end{aligned}
$$

From (4) and (5) we get

$$
\pi(x, 0,0)=\bar{r} \frac{1-x^{K}}{1-x}
$$

and

$$
\pi(x, y, 0)=\bar{r} \frac{1-x^{K}}{1-x}+y r \frac{1-x^{K}}{1-x}+y x^{K} .
$$


In (24) and (25), as well as in the rest of the paper, we understand that for $x=1$ and for all $\mathrm{K},\left(1-x^{K}\right) /(1-x)=K$. Thus we obtain

$$
\begin{aligned}
\pi(x, y, z)-\pi(x, 0, z)=y x^{K}+r \frac{1-x^{K}}{1-x} y+\bar{r} \frac{\rho \alpha^{2}}{1-\alpha x} \frac{z}{\alpha x}[\pi(x, y, z)-\pi(x, 0, z)] \\
\quad-\frac{\bar{r} \rho \alpha^{2}}{1-\alpha x}(\alpha x)^{K} z\left[\pi\left(\alpha^{-1}, y, z\right)-\pi\left(\alpha^{-1}, 0, z\right)\right]-\frac{\bar{r} \rho \alpha^{2}}{1-\alpha x}\left(\frac{1}{\alpha x}-(\alpha x)^{K}\right) z[\pi(0, y, z)-\pi(0,0, z)] \\
+\bar{r} \alpha \frac{1-(\alpha x)^{K}}{1-\alpha x} z[\pi(0, y, z)-\pi(0,0, z)]+\alpha \rho(\alpha x)^{K} z y\left[\pi\left(\alpha^{-1}, y, z\right)+\frac{1}{\rho} \pi(0, y, z)\right] \\
+\frac{r \rho \alpha}{1-\alpha x} y z\left(\pi(x, y, z)-(\alpha x)^{K} \pi\left(\alpha^{-1}, y, z\right)\right)+r \alpha \frac{1-(\alpha x)^{K}}{1-\alpha x} y z \pi(0, y, z) \\
=\quad y x^{K}+r \frac{1-x^{K}}{1-x} y+\bar{r} \frac{\rho \alpha^{2} z}{(1-\alpha x) \alpha x}[\pi(x, y, z)-\pi(x, 0, z)]+\frac{r \rho \alpha y z}{1-\alpha x} \pi(x, y, z) \\
+\rho \alpha(\alpha x)^{K}\left(y-\frac{(\bar{r} \alpha+r y)}{1-\alpha x}\right) z\left[\pi\left(\alpha^{-1}, y, z\right)+\frac{1}{\rho} \pi(0, y, z)\right] \\
+\frac{\bar{r} \alpha^{2}(x-\rho)}{(1-\alpha x) \alpha x} z[\pi(0, y, z)-\pi(0,0, z)]+\frac{\bar{r} \rho \alpha^{2}(\alpha x)^{K}}{1-\alpha x} z\left[\pi\left(\alpha^{-1}, 0, z\right)+\frac{1}{\rho} \pi(0,0, z)\right] \\
+\frac{r \alpha y z}{1-\alpha x}(\alpha x)^{K} \pi(0, y, z) .
\end{aligned}
$$

We note that in order to establish the proof of Proposition 1, it follows from (3) that it suffices to obtain $\pi(x, y, z)$ at $x=\rho$, since

$$
q(y, z)=R_{K} \pi(\rho, y, z) .
$$

From (26), we have

$$
\begin{aligned}
& {[\pi(\rho, y, z)-\pi(\rho, 0, z)](1-(\bar{r}+r \rho y) z)=y \rho^{K}+r \frac{1-\rho^{K}}{1-\rho} y} \\
& \quad+z\left(y-\bar{r}-\frac{r y}{\alpha}\right)(\rho \alpha)^{K+1}\left[\pi\left(\alpha^{-1}, y, z\right)+\frac{1}{\rho} \pi(0, y, z)\right] \\
& \quad+z \bar{r}(\rho \alpha)^{K+1}\left[\pi\left(\alpha^{-1}, 0, z\right)+\frac{1}{\rho} \pi(0,0, z)\right]+r \rho y z\left[\pi(\rho, 0, z)+\frac{(\alpha \rho)^{K}}{\rho} \pi(0, y, z)\right] .
\end{aligned}
$$

To compute the function $\pi(\rho, y, z)$ it suffices to compute the functions in the square brackets as well as $\pi(\rho, 0, z)$. To do that, we first compute $\pi_{0, n}$ by proceeding in the same manner as 
in (22). Since $P_{K}^{a}(0, n)=0$ we have for $n \geq 2$,

$$
\begin{aligned}
\pi_{0, n}(x)= & \bar{r} \frac{\rho \alpha^{2}}{1-\alpha x} \frac{1}{\alpha x} \pi_{0, n-1}(x)-\bar{r} \frac{\rho \alpha^{2}}{1-\alpha x}(\alpha x)^{K} \pi_{0, n-1}\left(\alpha^{-1}\right) \\
& +\bar{r} \alpha \frac{1-(\alpha x)^{K}}{1-\alpha x} \pi_{0, n-1}(0)-\bar{r} \frac{\rho \alpha^{2}}{1-\alpha x}\left(\frac{1}{\alpha x}-(\alpha x)^{K}\right) \pi_{0, n-1}(0) .
\end{aligned}
$$

By taking the generating function of both sides of the above equation and substituting (24), we can write

$$
\begin{aligned}
(1- & \alpha x) \alpha x \pi(x, 0, z)=\bar{r} \frac{1-x^{K}}{1-x}(1-\alpha x) \alpha x+\bar{r} \rho \alpha^{2} z \pi(x, 0, z) \\
& -\bar{r} \rho \alpha^{2}(\alpha x)^{K+1} z\left[\pi\left(\alpha^{-1}, 0, z\right)+\frac{1}{\rho} \pi(0,0, z)\right]+\bar{r} \alpha^{2}(x-\rho) z \pi(0,0, z) .
\end{aligned}
$$

From (26), we have

$$
\begin{aligned}
& \left((1-\alpha x) \alpha x-\rho \alpha^{2} \bar{r} z\right)[\pi(x, y, z)-\pi(x, 0, z)] \\
& =\quad(1-\alpha x) \alpha y x^{K+1}+(1-\alpha x) \alpha x r \frac{1-x^{K}}{1-x} y \\
& \quad+z \rho \alpha(\alpha x)^{K+1}\left[(y(1-\alpha x)-(\bar{r} \alpha+r y)] \times\left[\pi\left(\alpha^{-1}, y, z\right)+\frac{1}{\rho} \pi(0, y, z)\right]\right. \\
& \quad+\bar{r} \rho \alpha^{2}(\alpha x)^{K+1} z\left[\pi\left(\alpha^{-1}, 0, z\right)+\frac{1}{\rho} \pi(0,0, z)\right]+\alpha^{2} r \rho x y z \pi(x, y, z) \\
& \quad+\alpha^{2} \bar{r}(x-\rho) z[\pi(0, y, z)-\pi(0,0, z)]+\alpha^{2} r x y z(\alpha x)^{K} \pi(0, y, z) .
\end{aligned}
$$

Substituting (28) in (29) yields

$$
\begin{aligned}
& \left((1-\alpha x) \alpha x-\rho \alpha^{2}(\bar{r} z+r x y z)\right) \pi(x, y, z) \\
& \quad=(1-\alpha x) \alpha y x^{K+1}+(1-\alpha x) \alpha x(r y+\bar{r}) \frac{1-x^{K}}{1-x} \\
& +z \rho \alpha(\alpha x)^{K+1}(y(\bar{r}-\alpha x)-\bar{r} \alpha) \times\left[\pi\left(\alpha^{-1}, y, z\right)+\frac{1}{\rho} \pi(0, y, z)\right] \\
& \quad+z \alpha^{2}\left(\bar{r}(x-\rho)+r x y(\alpha x)^{K}\right) \pi(0, y, z)
\end{aligned}
$$

For each $i=1,2$, when $x=x_{i}(y, z)$, the term that multiplies $\pi(x, y, z)$ in the left hand side of equation (30) vanishes. Since $\pi(x, y, z)$ is polynomial in $x$ and therefore analytic in $x$, the left 
hand side of (30) vanishes at $x=x_{i}(y, z)$. Thus by substituting $x_{i}$ for $x$ into (30), we obtain

two equations (Equation (8)) with two unknowns: $A(y, z)=\left[\pi\left(\alpha^{-1}, y, z\right)+\frac{1}{\rho} \pi(0, y, z)\right]$ and $B(y, z)=\pi(0, y, z)$. Equation (7) of the proposition, finally, follows from (30) with $x=\rho$ and (27).

\section{References}

[1] E. Altman, C. Barakat and V. M. Ramos R., "Queueing Analysis of Simple FEC schemes for IP Telephony", Computer Networks, Vol. 39, No. 2, pp. 185-206, June 2002.

[2] E. N. Gilbert, "Capacity of a Burst-Noise Channel",Bell Systems Technical Journal, Vol. 39, pp. 1253-1265, Sept 1960

[3] E. Altman, K. Avrachenkov, C. Barakat, P. Dube, "TCP Over a Multi-State Markovian Path", In Proc. of Performance and QoS of Next Generation Networking, P \& Q Net2000, Nagoya, Japan, Nov. 2000.

[4] O. Ait-Hellal, E. Altman, A. Jean-Marie, I. A. Kurkova, "On Loss Probabilities in Presence of Redundant Packets and Several Traffic Sources", Performance Evaluation, 36-37, pp. 485-518, 1999.

[5] I. Cidon, A. Khamisy and M. Sidi, "Analysis of Packet Loss Process in High-Speed Networks", IEEE Trans. onInformation Theory, Vol. 39, No. 1, pp. 98-108, 1993.

[6] L. Takacs, "Combinatorial Methods in the Theory of Stochastic Processes", New York, Wiley, 1967.

[7] N. Shacham and P. McKenney, "Packet Recovery in High-Speed Networks Using Coding and Buffer Management", INFOCOM '90, San Francisco, CA, pp. 124-131, 1990.

[8] I. Cidon, R. Guerin, I. Kessler and A. Khamisy, "Analysis of a Statistical Multiplexer with Generalized Periodic Sources", Queueing Systems, 20, pp. 139-169, 1995.

[9] O. Gurewitz, M. Sidi and I. Cidon, "The Ballot Theorem Strikes Again: Packet Loss Process Distribution", IEEE Transactions on Information Theory, 46, No. 7, Nov 2000.

[10] N. Cem Oğuz and E. Ayanoğlu, "Performance Analysis of Two-Level Forward Error Correction for Lost Cell Recovery in ATM Networks", In Proc. of INFOCOM '95, pp. 6b.3.1-6b.3.10, 1995.

[11] E. Altman and A. Jean-Marie, "Loss probabilities for Messages with Redundant Packets Feeding a Finite Buffer", IEEE Journal of Selected Areas in Communications, , Vol. 16, No. 5, pp. 779-787, 1998. 